\begin{tabular}{|c|c|c|c|c|c|c|}
\hline \multirow{4}{*}{ Impact Factor: } & ISRA (India) & $=4.971$ & SIS (USA) & $=0.912$ & ICV (Poland) & $=6.630$ \\
\hline & ISI (Dubai, UAE & $=0.829$ & РИНЦ (Russia) & $=0.126$ & PIF (India) & $=1.940$ \\
\hline & GIF (Australia) & $=0.564$ & ESJI (KZ) & $=8.716$ & IBI (India) & $=4.260$ \\
\hline & JIF & $=1.500$ & SJIF (Morocco) & $=5.667$ & OAJI (USA) & $=0.350$ \\
\hline
\end{tabular}

\section{SOI: 1.1/TAS DOI: $10.15863 /$ TAS \\ International Scientific Journal Theoretical \& Applied Science}

p-ISSN: 2308-4944 (print)

e-ISSN: 2409-0085 (online)

Year: 2020

Issue: 03

Volume: 83

Published: 30.03 .2020

\section{http://T-Science.org}

QR - Issue

QR - Article
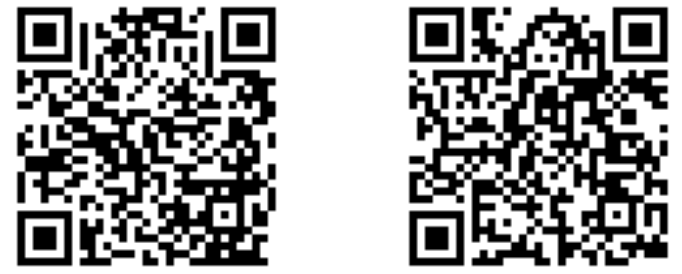

N.N. Tkhelidze

Akaki Tsereteli State University Ph.D., Engineering Sciences PhD. Associate Professor, Head of Department Design and Technology

M. G. Grdzelidze

Akaki Tsereteli State University Ph.D., Professor of the Department "Design and Technology", Dean of Engineering-Technological Faculty

Georgia, Kutaisi

I. Charkviani

Akaki Tsereteli State University

Ph.D., Georgia, Kutaisi

\title{
QUALITY ASSESSMENT IN MANUFACTURE OF CLOTHING
}

\author{
Abstract: In order to improve the consumer properties and visual quality of product, it is necessary to activate \\ the enterprise quality management system. The article considers the quality assessment during performing the \\ technological operations of sewing. \\ Product quality assessment methods allow us for checking the consumer properties of finished goods and valuing \\ the losses, depending on the type of materials and product failures, which in turn should be used to prevent defects \\ in production. Improvements in the quality assessment methods at all stages of manufacture of clothing is a \\ prerequisite for producing competitive products and consequently for increasing efficiency of production. \\ Key words: the cloth, clothes, Quality, defects, Quality control. \\ Language: Russian \\ Citation: Tkhelidze, N. N., Grdzelidze, M. G., \& Charkviani, I. (2020). Quality assessment in manufacture of \\ clothing. ISJ Theoretical \& Applied Science, 03 (83), 337-341. \\ Soi: http://s-o-i.org/1.1/TAS-03-83-62 Doi: crossef https://dx.doi.org/10.15863/TAS.2020.03.83.62 \\ Scopus ASCC: 2209.
}

\section{ОЦЕНКА КАЧЕСТВА ПРИ ИЗГОТОВЛЕНИИ ОДЕЖДЫ}

Аннотация: Для усовершенствования потребительских свойств и визуального качества изделия, необходимо активировать систему управления качеством предприятия. В статье рассматривается оценка качества технологии выполнения швейных операций. Методы оценки качества продукиии позволяют нам 6 зависимости от материалов и вида дефектов, проверить потребительские свойства готового изделия и оценивать их потери, который в свою очередь должен быть использован для профилактики дефектов в производстве. Совершенствование методов оченки качества на всех этапах производства одежды является обязательным условием для изготовления конкурентоспособного изделия и, следовательно, для повышения эффективности производства.

Ключевые слова: ткань, одежда, качество, дефекты, оценка качества.

\section{Введение}

В рыночной экономике наибольшее внимание уделяется проблеме качества из-за существования

конкурентной среды.

Современные методы контроля качества продукции приобретают все большее значение и позволяют добиться высокой стабильности качественных показателей при минимальных 


\begin{tabular}{|c|c|c|c|c|c|c|}
\hline \multirow{4}{*}{ Impact Factor: } & ISRA (India) & $=4.971$ & SIS (USA) & $=0.912$ & ICV (Poland) & $=6.630$ \\
\hline & ISI (Dubai, UAE & $=0.829$ & РИНЦ (Russia & $=0.126$ & PIF (India) & $=1.940$ \\
\hline & GIF (Australia) & $=0.564$ & ESJI (KZ) & $=8.716$ & IBI (India) & $=4.260$ \\
\hline & JIF & $=1.500$ & SJIF (Morocec & $=5.667$ & OAJI (USA) & $=0.350$ \\
\hline
\end{tabular}

затратах. Эффективная система контроля позволяет своевременно и преднамеренно влиять на качество производимой продукции, избегать всех возможных недостатков и сбоев в работе и обеспечить минимальными расходами оперативное воздействие и устранение дефектов[1-4].

Положительные эффекты текущего контроля качества могут быть определены на стадиях обработки продукта, производства, эксплуатации (клиент) и восстановления (ремонт). Экспертиза дефектов продукции легкой промышленности отличаются в зависимости от вида товаров. Качество экспертизы материалов зависит от вида используемых материалов. Суммарные штрафные баллы принимаются путем суммирования физикомеханических характеристик, оценки внешних и локальных дефектов. Например, в случае тканей: в тканях I степени не допускаются заметные дефекты, в тканях II степени допустимо не более одного распространнённого дефекта $[6,8]$

Количество штрафных баллов местного дефекта рассчитывается по условной длине:

$$
Б_{f}=\sum B \frac{L_{g}}{L}
$$

где: $\sum S$ - сумма штрафных баллов в материале для дефекта внешнего вида материала длиной $L$;

$L_{g}$ - условная длина ткани в зависимости от ширины ткани.

Допустимое количество дефектов материалов, используемых на предприятиях легкой промышленности, определяются стандартом. Например, количество дефектов на условной площади ткани $3 \mathrm{M}^{2}$ рассчитывается следующим образом:

$$
n_{f}=n_{a} \frac{3 \cdot 10^{3}}{L S}
$$

где: $n_{a}$ - количество фактических дефектов;

$$
\begin{aligned}
& L \text { - длина; } \\
& S \text {-площадь ткани; }
\end{aligned}
$$

\section{Объекты и методы исследования}

Объектами исследования являются материалы, применяемые в одежды и в одежды.
Качество готовой продукции определяется тремя методами - внешним осмотром, линейным измерением и лабораторным обследованием. Внешний осмотр производится на поверхности или со стороны подкладки, для которого производят сравнивание и измерение отдельных деталей, определение частоты стежков путем подсчета его количества на определенном участке $[5,7]$

При оценке качества обуви ее сложность определяется множеством деталей и сложной и разнообразной технологией сбора изделии. Обувные материалы отличаются в зависимости от его расположения и назначения в изделии. Процесс сбора изделии отличается в зависимости от используемых методов и средств и зависит от точности нормативов выполнения технологической операции. По этому оценка и контроль качества обуви должны проводиться на всех этапах - от приёма материала до получения готового изделия. Особенно важным является качество связи заготовки с подошвом и деталей заготовки. Так как они напрямую связаны с сохранением целостности изделия во время эксплуатации.

\section{Постановка задачи}

Целью данной статьи является определение качества одежды путём лабораторного исследования.

\section{Результаты и их обсуждения}

Для определения качества путём лабораторного исследования, выбор образца производится непосредственно экспертом с разных мест изделии и количество образцов зависит от величины партии товара. Контролью надлежат: ввозимое в производство сырье, основные и вспомогательные матерьялы, полуфабрикаты, комплектующие изделия, производственные изделия, детали, сборочные единицы, оборудования, снаряжения, технологические процессы изготовления продукции.

Проведена оценка качества операций по пошиву одежды в готовом изделии и была составлена соответствующая схема (табл. 1; рис. 1, 2).

Табл. 1. Виды дефектов в процессе выполнения технологических операций

\begin{tabular}{|l|lc|c|c|c|c|}
\hline$\#$ & \multicolumn{1}{|l}{ Показатели вида дефектов } & $\begin{array}{l}\text { Количество } \\
\text { дефектов } \\
\text { образцов }\end{array}$ & $\begin{array}{c}\text { Растущая } \\
\text { сумма } \Sigma \mathbf{n}_{\mathbf{i}}\end{array}$ & $\begin{array}{c}\Delta \mathbf{I}=\mathbf{n}_{\mathbf{i}} / \Sigma \mathbf{n}_{\mathbf{i}} \\
* \mathbf{1 0 0 \%}\end{array}$ & $\begin{array}{c}\text { Растущая } \\
\text { сумма } \Sigma \mathbf{n}_{\mathbf{i}} \%\end{array}$ \\
\hline & $\begin{array}{l}\text { Неправильный сгиб лацкана } \\
\text { воротника }\end{array}$ & 3 & 3 & 5 & 5 \\
\hline
\end{tabular}




\begin{tabular}{|c|c|c|c|c|c|c|}
\hline \multirow{4}{*}{ Impact Factor: } & ISRA (India) & $=4.971$ & SIS (USA) & $=0.912$ & ICV (Poland) & $=6.630$ \\
\hline & ISI (Dubai, UAE & $=0.829$ & РИНЦ (Russia) & $=0.126$ & PIF (India) & $=1.940$ \\
\hline & GIF (Australia) & $=0.564$ & ESJI (KZ) & $=8.716$ & IBI (India) & $=4.260$ \\
\hline & JIF & $=1.500$ & SJIF (Morocco) & $=5.667$ & OAJI (USA) & $=0.350$ \\
\hline
\end{tabular}

\begin{tabular}{|l|l|c|c|c|c|}
\hline & Искривление закрепления шва & 9 & 12 & 14 & 19 \\
\hline & Разность длин бортов & 2 & 14 & 3 & 21 \\
\hline $\begin{array}{l}\text { Неровное распределение рукава в } \\
\text { срезе }\end{array}$ & 2 & 16 & 3 & 24 \\
\hline $\begin{array}{l}\text { Рамка карманного среза } \\
\text { растянута }\end{array}$ & 5 & 21 & 8 & 32 \\
\hline $\begin{array}{l}\text { Подкладка талии брюк видна на } \\
\text { внешней стороне }\end{array}$ & 2 & 23 & 3 & 35 \\
\hline $\begin{array}{l}\text { Нижный срез брюк не } \\
\text { разработана тесмой }\end{array}$ & 3 & 26 & 5 & 40 \\
\hline & $\begin{array}{l}\text { Деформация прокладочного } \\
\text { материала }\end{array}$ & 7 & 33 & 11 & 51 \\
\hline & Отклонение отделочного шва & 8 & 41 & 12 & 62 \\
\hline & Обрезка ниток в шве & 4 & 45 & 6 & 68 \\
\hline & Утолщение ниток & 3 & 48 & 5 & 65 \\
\hline & Близна & 2 & 50 & 3 & 68 \\
\hline & Несовпадение картинки & 3 & 53 & 5 & 73 \\
\hline & Некрашенные места & 4 & 57 & 6 & 79 \\
\hline & Пятна & 8 & 65 & 11 & 100 \\
\hline
\end{tabular}

Диаграмма (рис. 1) показывает, что наиболее распространенными являются: 1) дефект отсутствия закрепленной строчки (9); 2) пятна и отклонения декоративной строчки (8); 3) Деформация материала прокладки (7).

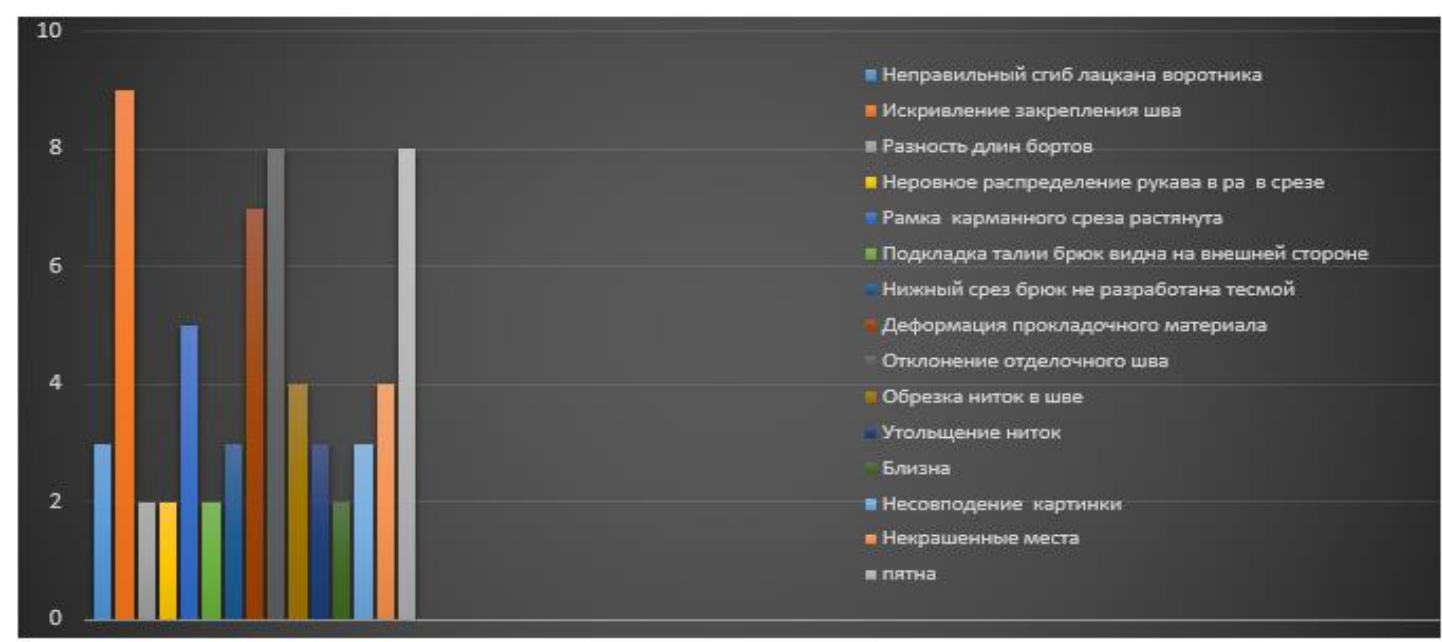

Рис. 1. Количество дефектов образца

Диаграмма потеря качества в совокупности показана на рис. 2 


\begin{tabular}{|c|c|c|c|c|c|c|}
\hline \multirow{4}{*}{ Impact Factor: } & ISRA (India) & $=4.971$ & SIS (USA) & $=0.912$ & ICV (Poland) & $=6.630$ \\
\hline & ISI (Dubai, UAE & $=0.829$ & РИНЦ (Russia & $=0.126$ & PIF (India) & $=1.940$ \\
\hline & GIF (Australia) & $=0.564$ & ESJI (KZ) & $=8.716$ & IBI (India) & $=4.260$ \\
\hline & JIF & $=1.500$ & SJIF (Morocec & $=5.667$ & OAJI (USA) & $=0.350$ \\
\hline
\end{tabular}

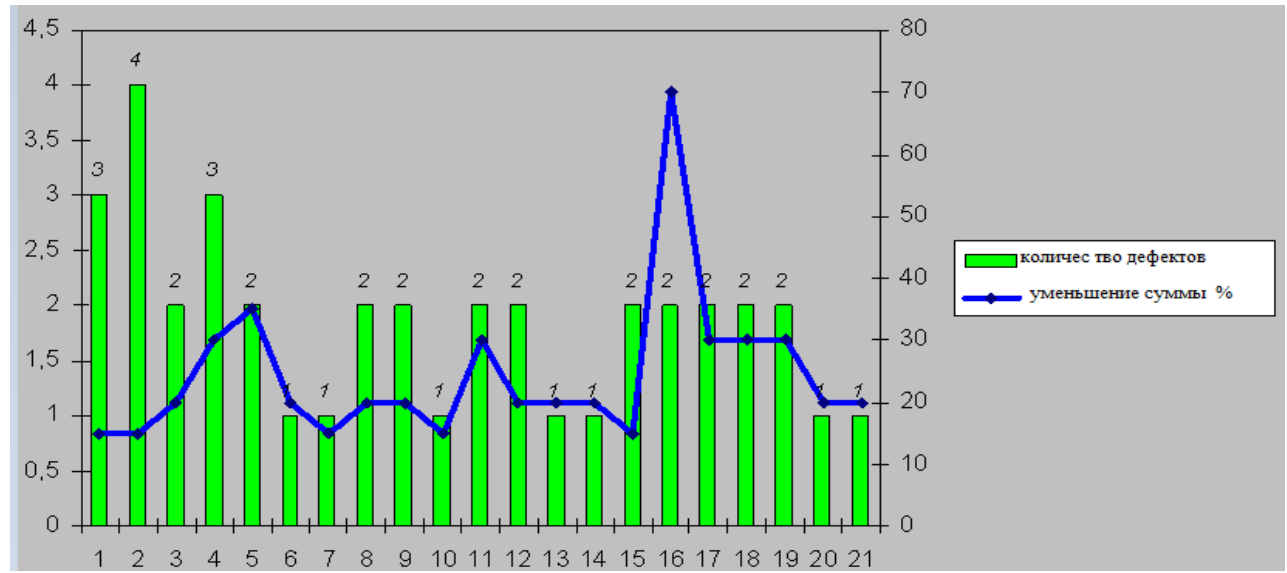

Рис. 2. Потеря качества в совокупности в зависимости от вида дефекта.

В исследованиях, проведённых для оценки качества изделии легкой промышленности (здесь рассматриваются результаты исследования качества одежды), результаты сравнения по видам дефекта показаны в таблице 2, а распределение дефектов по потребительским свойством показано в таблице 3, на основе которых были построены диаграммы распределения (рисунок 3)[9,10].

Табл. 2. Виды дефектов

\begin{tabular}{|c|c|c|c|c|c|}
\hline \# & $\begin{array}{c}\text { Вид дефектов готовой } \\
\text { продукции }\end{array}$ & $\begin{array}{l}\text { Количество } \\
\text { дефектов } \mathbf{n}_{\mathbf{i}}\end{array}$ & $\begin{array}{l}\text { Растущая } \\
\text { сумма } \Sigma \mathrm{n}_{\mathrm{i}}\end{array}$ & $\Delta I=n_{i} / \Sigma n_{i} * 100 \%$ & $\begin{array}{c}\text { Растущая } \\
\text { сумма } \Sigma \mathbf{n}_{\mathbf{i}} \%\end{array}$ \\
\hline 1 & Виды внешних дефектов & 32 & 32 & 47 & 47 \\
\hline 2 & $\begin{array}{l}\text { Несоответствие с эталоном- } \\
\text { образцом }\end{array}$ & 1 & 33 & 2 & 49 \\
\hline 3 & $\begin{array}{l}\text { Низкие } \\
\text { механические показатели }\end{array}$ & 9 & 42 & 14 & 63 \\
\hline 4 & $\begin{array}{l}\text { Нарушение маркировки и } \\
\text { упаковки }\end{array}$ & 18 & 60 & 26 & 89 \\
\hline 5 & Разное & 7 & 67 & 11 & 100 \\
\hline
\end{tabular}

Табл. 3. Виды дефектов по потребительским свойствам

\begin{tabular}{|c|c|c|c|c|c|}
\hline \# & $\begin{array}{c}\text { Виды дефектов по } \\
\text { потребительским свойствам }\end{array}$ & $\begin{array}{c}\text { Количество } \\
\text { образцов с } \\
\text { уменшением } \\
\text { качества }\end{array}$ & $\begin{array}{l}\text { Растущая } \\
\text { сумма } \Sigma \mathbf{n}_{\mathbf{i}}\end{array}$ & $\begin{array}{l}\Delta \mathrm{I}=\mathrm{n}_{\mathrm{i}} / \Sigma \mathrm{n}_{\mathrm{i}} \\
\quad * \mathbf{1 0 0 \%}\end{array}$ & $\begin{array}{c}\text { Растущая } \\
\text { сумма } \Sigma \mathrm{n}_{\mathrm{i}} \\
\%\end{array}$ \\
\hline 1 & Волокнянный состав & 1 & 1 & 5 & 5 \\
\hline 2 & Толщина ткани & 3 & 4 & 14 & 19 \\
\hline 3 & $\begin{array}{l}\text { Твердость, } \\
\text { драпируемость }\end{array}$ & 2 & 6 & 10 & 29 \\
\hline 4 & Усадка & 5 & 11 & 24 & 53 \\
\hline 5 & Электростатистический заряд & 4 & 15 & 19 & 72 \\
\hline 6 & Воздухопроницаемость & 6 & 21 & 28 & 100 \\
\hline
\end{tabular}




\begin{tabular}{|c|c|c|c|c|c|c|}
\hline \multirow{4}{*}{ Impact Factor: } & ISRA (India) & $=4.971$ & SIS (USA) & $=0.912$ & ICV (Poland) & $=6.630$ \\
\hline & ISI (Dubai, UAE & $=0.829$ & РИНЦ (Russia & $=0.126$ & PIF (India) & $=1.940$ \\
\hline & GIF (Australia) & $=0.564$ & ESJI (KZ) & $=8.716$ & IBI (India) & $=4.260$ \\
\hline & JIF & $=1.500$ & SJIF (Morocce & $=5.667$ & OAJI (USA) & $=0.350$ \\
\hline
\end{tabular}
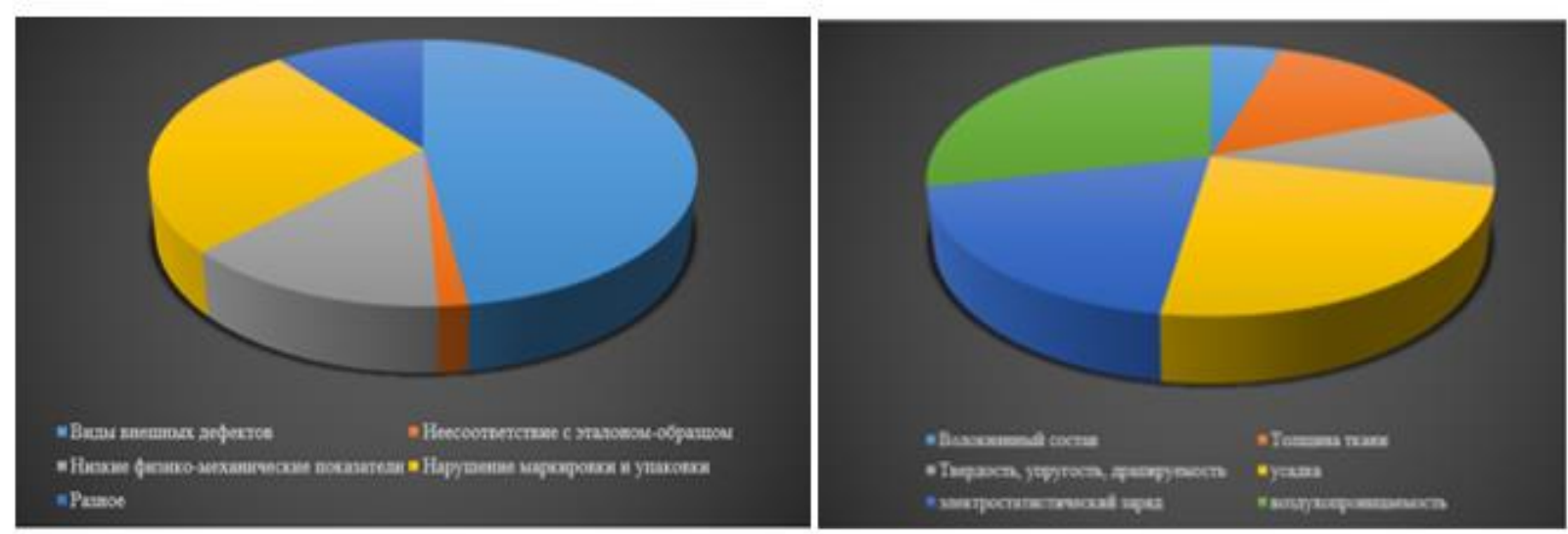

Рис. 3. Виды дефектов готового изделия

\section{Выводы:}

Как видно из диаграммы, внешние дефекты в готовой продукции представлены в значительной степени, что однозначно указывает на то, что предприятию придется потратить некоторые материальные затраты на улучшение потребительских свойств и визуального качества изделия, в частности, активация систем входящего контроля предприятия, на контроль подготовительных операции, на текущий контроль производственного процесса и на заключительном этапе производства на контроль процесса отделки готового изделия - для производства обусловленно необходимостью и расходы, понесенные для этой цели, станут предпосылкой для экономии ресурсов.

\section{References:}

1. Solov'ev, A.N., \& Kirjuhin, S.M. (1987). Ocenka i prognozirovanie kachestva tekstil'nyh materialov. (p.268). Moscow: Legkaja i pishhevaja promyshlennost'.

2. Dodonkin, Ju.V., \& Kirjuhin, S.M. (2000). Asortimenti, svojstva i ocenka kachestva tkanej. (p.279). Moscow: Legkaja industrija.

3. Kirjuhin, S.M., \& Solovev, A.N. (1977). kontrol i upravlenie kachestvo tekstil'nyh materialov. (p.310). Moskva megkaja industrija.

4. Honsen, B.L. (2003). Kontrol' kachestva. (p.620). Moscow: «Progress».

5. Potulova, V.V. (1990). metody obespechenija kachestva produkcii. (p.57). Moscow: Izd-vo standartov.

6. Kukin, G.N., Solov'ev, A.N., \& Kobljakov, A.I. (1992). Tekstil'noe materialovedenie (tekstil'nye polotna i izdelija). (p.271). Moscow: Legprombytizdat.

7. Mhitarjan, L.S. (2008). «Defekty odezhdy: spravochnik». Izd-vo AST.

8. Buzov, B.A., et al. (2009). Materialovedenie shvejnogo proizvodstva. (p.322). Moscow.

9. Thelidze, N.N. (2012). Monografija "Issledovanie potrebitel'skih svojstv legkoj promyshlennosti”. Gruzija, ATSU.

10. Thelidze, N.N. (n.d.). Izuchenie novyh kriterii dlja opredelenija potrebitel'skij svojstv tekstil'nyh izdelii, mezhdunarodnaja konferencija. «Nauka i praktika: novyj uroven' integracii $\mathrm{V}$ sovremennom mire». SCOPE ACADEMIC HOUSE, UK, S Yorkshire, Sheffieldm. B\&M publishing. USA, San Francisco, California. ISBN 978-1-941655-726, DOI: http://doi.org/10.15350/UK_6/5 